\title{
SIMULAÇÃO COMPUTACIONAL DE NUCLEAÇÃO E CRESCIMENTO DE GRÃOS*
}

\section{Resumo}

A maneira com que acontece a nucleação interfere diretamente na forma dos contornos de grão e também nas propriedades do material em questão. Para visualizar melhor, pode-se utilizar uma célula cúbica, na qual é possível notar que em cada eixo há diferença de textura e densidade entre os grãos já desenvolvidos. Neste trabalho, foram empregadas duas vias de estudo a fim de obter os resultados desejados. A primeira delas é a nucleação homogênea dos grãos e a outra, a nucleação não homogênea, ambas com velocidade constante. Esse fenômeno pode ser visto durante a recristalização de grãos. Rios e Villa [6] criaram um método efetivo para analisar a cinética das transformações homogêneas e não homogêneas a partir do método de JMAK[1-5]. Para comparar método analítico foi desenvolvida uma simulação computacional com base no modelo analítico Rios e Villa [6], foi utilizado a metodologia do Cone Causal (método estocástico). Os gráficos gerados confirmam a teoria de Rios e Villa [6] para esses casos particulares e comparam os resultados obtidos com o conceito de JMAK [1-5].

Palavras-chave: nucleação; homogênea; recristalização; simulação computacional.

\section{Abstract}

\section{NUCLEATION AND GROWTH GRAINS COMPUTATIONAL SIMULATION}

The way that the nucleation has created, that interferes directly with the shape of the grain boundaries, also with the material's properties. To the better visualization, we can use a cubic cell, which it's possible to notice that in each axis there is difference of texture and density among the developed grains. In this work, two study routes were used in order to obtain the desired results. The first one is the grains homogeneous nucleation and the other, is the non-homogeneous nucleation, both with constant velocity. This phenomenon can be seen during the grain recrystallization. Rios and Villa[1-5] have created an effective method to analyze the kinetics of homogeneous and non-homogeneous transformations from the JMAK[6] method. To compare the analytical method, a computational simulation was developed based on the Rios and Villa analytical model, using the Causal Cone methodology (stochastic method). The generated graphs confirm the Rios and Villa[6] theory for these particular cases and compare the results obtained with the JMAK concept[1-5].

Keywords: nucleation; homogeneous; recrystallization; computer simulation

1 Engenharia Metalúrgica, Graduando, estudante, Universidade Federal Fluminense, Núcleo de Modelamento Microestrutural, UFF-EEIMVR, Sala C87, Volta Redonda, RJ, Brasil.

2 Engenharia Metalúrgica, Mestre, estudante, Universidade Federal Fluminense, Núcleo de Modelamento Microestrutural, UFF-EEIMVR, Sala C87, Volta Redonda, RJ, Brasil.

3 Engenharia Metalúrgica, Doutorando, estudante, Universidade Federal Fluminense, Núcleo de Modelamento Microestrutural, UFF-EEIMVR, Sala C87, Volta Redonda, RJ, Brasil.

4 Engenharia Metalúrgica, Doutorando, estudante, Universidade Federal Fluminense, Núcleo de Modelamento Microestrutural, UFF-EEIMVR, Sala C87, Volta Redonda, RJ, Brasil.

5 Engenharia Metalúrgica, Graduando, estudante, Universidade Federal Fluminense, Núcleo de Modelamento Microestrutural, UFF-EEIMVR, Sala C87, Volta Redonda, RJ, Brasil.

6 Engenharia Metalúrgica, Doutor, professor, Universidade Federal Fluminense, Núcleo de Modelamento Microestrutural, UFF-EEIMVR, Sala C87, Volta Redonda, RJ, Brasil.

7 Engenharia Metalúrgica, Doutor, professor, Universidade Federal Fluminense, Núcleo de Modelamento Microestrutural, UFF-EEIMVR, Sala C87, Volta Redonda, RJ, Brasil. 


\section{INTRODUÇÃO}

Utilizada em vários campos de pesquisa básica e científica, a simulação computacional é uma ferramenta poderosa na área de engenharia. Neste caso a simulação computacional foi aplicada em transformação de fase em estudos de engenharia metalúrgica. As transformações de fase no estado sólido normalmente ocorrem por processo de nucleação e crescimento, que pode ser interpretado quantitativamente e qualitativamente por modelos analíticos, numéricos e experimentais. Os modelos analíticos básicos da cinética formal utilizados para o estudo de transformações de fases heterogêneas, conhecida há quase 80 anos iniciaram com Johnson-Mehl[1], Avrami[2-4] e Kolmogorov[5]. Esse modelo tem sido amplamente utilizado para tratar transformações de fase e também, mais comumente, o fenômeno da recristalização. Essa teoria foi desenvolvida a partir de suposições como a nucleação aleatória e homogênea, crescimento com velocidade constante, forma dos grãos esférica e homogeneidade energética na matriz deformada.

A solução analítica de JMAK[1-5]obtida considera os núcleos com formato esférico, a nucleação por saturação de sítios, ou seja, considera que todos os núcleos surgiram, nos instantes iniciais da reação, onde $N_{v}$ é o número de núcleos por unidade de volume e $G$ é a velocidade de crescimento. Resultando na conhecida (Equação 1):

$V_{V}(t)=1-\exp \left(-\frac{4 \pi}{3} N_{V} G^{3} t^{3}\right)$

Apesar da teoria de JMAK [1-5] ser amplamente empregada, ela possui limitações, que foram feitas para a dedução destas equações, a saber:

- As transformações devem ocorrer em um meio infinito;

- Os núcleos devem estar distribuídos de forma uniformemente aleatória no espaço;

- Os núcleos possuem uma forma geométrica similar e seu crescimento irá cessar quando ocorrer a colisão entre as regiões transformadas.

- O crescimento deve ser isotrópico.

Essas restrições nem sempre são satisfeitas em sistemas reais, o que causa um desvio da cinética de JMAK[1-5].

Entretanto, em diversas situações há a possibilidade dessa nucleação não ser uniforme. Por exemplo, a nucleação da recristalização em uma chapa deformada de tal forma que existe um gradiente de deformação entre a superfície superior e inferior. Nesse caso, a nucleação é mais abundante na região mais deformada e menos abundante na região menos deformada.

Recentemente, Rios e Villa [6] revisaram os trabalhos de JMAK e utilizando a metodologia da geometria Estocástica trataram a disposição espacial dos sítios de 
nucleação como um processo de ponto. Mais especificamente, como um processo de ponto de Poisson que pode ser homogêneo ou não homogêneo.

O processo de ponto de Poisson não homogêneo sera dependete da fração volumetrica dependente da posição $V v(t, p)$, a fração volumetrica estendida, onde $\mathrm{p}=$ $(\mathrm{x}, \mathrm{y}, \mathrm{z})$, é a coordenada espacial .Assim , arrelação entre a fração volumetrica e a fração volumetrica estendida , tornou-se uma relação entre a densidade media do volume e a densidade volume extendido :

$$
V_{V}(t, x)=1-\exp \left(-V_{E}(t, p)\right)
$$

Usando esta metodologia, foi possível ampliar significativamente o escopo de soluções analíticas disponíveis. Um exemplo dos resultados obtidos pelos Rios e Villa é uma equação para a nucleação por saturação de sitios não homogenea e dada por :

$$
\begin{aligned}
& \lambda(\mathrm{p})=\mathrm{mz}+\mathrm{n} \\
& V_{V}(t, p)=1-\exp \left(-\frac{4 \pi}{3} \lambda(p) G^{3} t^{3}\right)
\end{aligned}
$$

Onde a (equação 4) permite modelar processos de Poisson homogêneos se $\lambda$ independe de $\mathrm{p}$ ou não homogêneos se $\lambda$ depende de $\mathrm{p}$ da posição no espaço.

\section{MATERIAIS E MÉTODOS}

Neste trabalho utilizou-se a metodologia do Cone Causal para a simulação das reações com nucleação e crescimento, que ocorrem de forma homogênea e não homogênea. Foi utilizado um programa computacional escrito em linguagem Fortran 2003, e compilado pelo Microsoft Visual Studio 2012. Utilizou-se paralelização em Open MP. A visualização das microestruturas transformadas foi realizada como programa Tecplot 360 B, os gráficos foram construídos como auxílio do programa Wolfram Mathematica $10 \AA$.

Em todos os casos simulou-se em uma matriz cúbica de $300^{3}$. Considerou-se uma quantidade de núcleos e velocidade constante em toda transformação. A nucleação se deu por saturação de sítios em todos os casos.

Para simular uma amostra infinita foram utilizadas condições de contorno periódicas para as direções $x, y$ e $z$ da matriz cúbica para nucleação homogênea sendo na direção z limitada para que os grãos não ultrapassem as condições de contorno na nucleação não homogênea.

Gerou-se em cada simulação, 150 números de núcleos, logo realizou-se as simulações distintas para cada caso. Com os dados gerados pelas simulações geram-se gráficos, assim compara-se as nucleações.

\subsection{Modelo computacional}

O código desenvolvido, neste trabalho, é o modelamento de processos que ocorrem por nucleação de núcleos que variam a quantidade dependente do seu posicionamento na matriz e taxa crescimento também vai dependendo do posicionamento. 
O código computacional inicial para simulação de reações com nucleação e crescimento em 3D foi desenvolvido pelo Assis [7] ao longo dos trabalhos desenvolvidos no Núcleo de Modelamento Microestrutural, código computacional possui duas formas distintas de crescimento o Autômato Celular e o Cone Causal Alves [8]. O código foi desenvolvido em ambiente Fortran e é utilizada a paralelização em Open MP.

O Programa inicia-se pela entrada de dados onde é preciso especificar os parâmetros que são usados na simulação, como por exemplo: tamanho de matriz, o modo de nucleação, a regra de transição (cone causal) e quantidade de núcleos iniciais. Após esta etapa, ocorre a formação da matriz, e a distribuição dos pontos onde serão alocados os núcleos inicias. Em seguida ocorre a nucleação, que ocorre por saturação de sítios.

Quando finalizada a nucleação o programa entra na sub-rotina de crescimento e é executado até a matriz tenha se transformado. Na saída de dados, o programa disponibiliza o tempo de reação fração volumétrica transformada $\left(V_{v}\right)$, área interfacial por unidade de volume (Sv), velocidade de crescimento de cada fase e o caminho microestrutural. Também geram outros dados que são utilizados para a visualização das microestruturas e sua evolução.

\subsection{Nucleação homogênea}

Este modelo de nucleação foi desenvolvido para simular o surgimento aleatório dos núcleos, dentro de uma matriz distinta de seus núcleos. É realizado um sorteio utilizando-se um código de gerador de números aleatórios para FORTRAN, este gerador escolhe aleatoriamente números que correspondem a posições dentro da matriz. Após cada núcleo ser alocado dentro da matriz de maneira aleatória, iniciou-se o crescimento dos mesmos. [9]

\subsection{Nucleação não-homogênea}

Nesta forma de nucleação os núcleos são distribuídos deforma não homogênea que pode variar em uma ou mais direções na matriz. Neste trabalho são mostrados e discutidos os resultados para variação de núcleos em uma única direção. Uma distribuição de núcleos com variação ao longo do eixo $Z$. A intensidade na direção $Z$ varia linearmente segundo a equação $\lambda(z)=m z+n$, onde $m$ e $n$ são constantes. [9]

\subsection{Cone causal}

Para o processo de crescimento na simulação computacional, neste trabalho optou-se pelo método do Cone Causal. A Figura 1, apresenta a teoria do Cone Causal em 2D. 


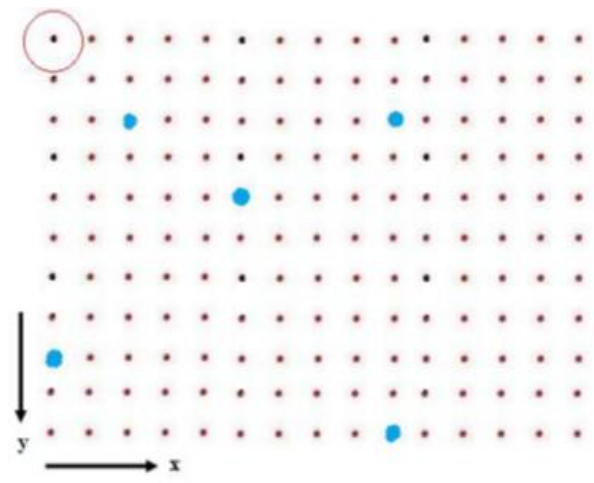

(a)

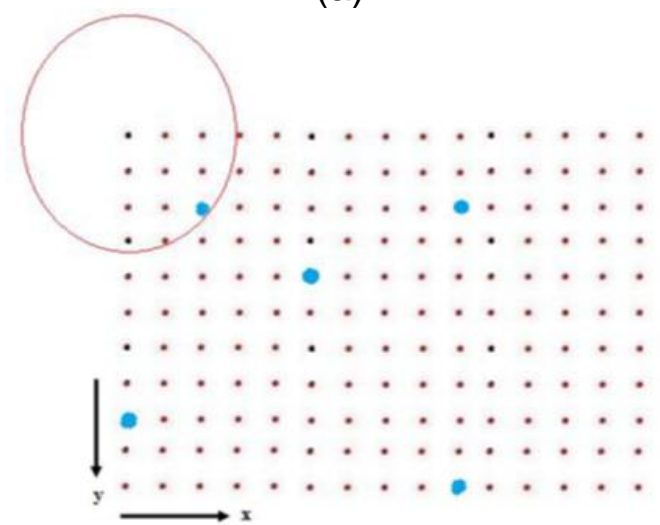

(c)

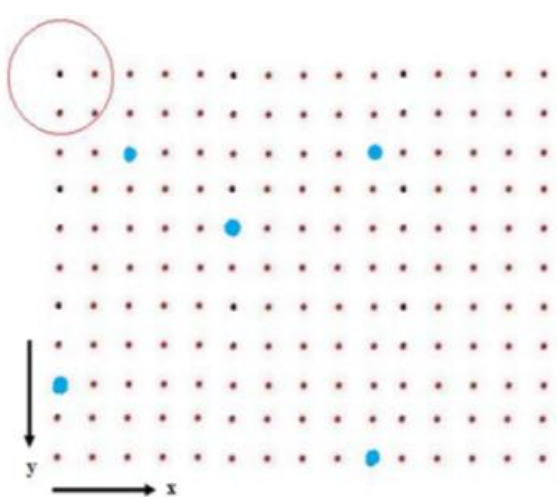

(b)

- pontos da matriz

- núcleos

Figura 1: Representação bidimensional da teoria do cone causal. (a) Tempo t1 (b) Tempo t2 (c) Tempo t3. Onde t3>t2>t1. [9]

O programa realiza iterações ao longo do tempo. Para cada iteração ocorre a transformação de um ponto qualquer sendo necessário haver pelo menos um núcleo dentro da esfera de raio $R$ que é centrada neste ponto qualquer. Dessa forma a esfera aumenta como tempo e à medida que o raio aumenta possibilidade de haver núcleos dentro da esfera é maior. Essa regra é realizada para todos os pontos da matriz a cada interação de tempo. 


\section{RESULTADOS E DISCUSSÃO}

A evolução microestrutural para nucleação homogênea para seis frações volumétricas distintas com número de 150 núcleos foi apresentada na Figura 2.

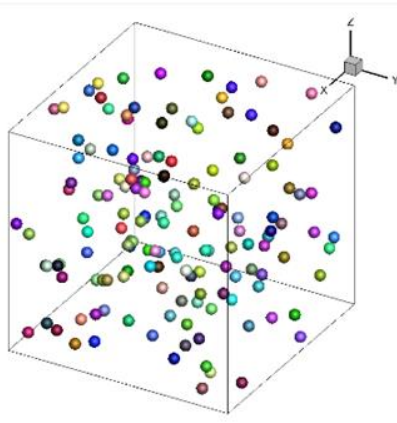

Inicial

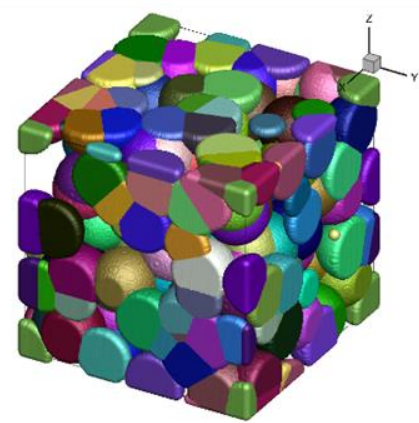

$50 \%$
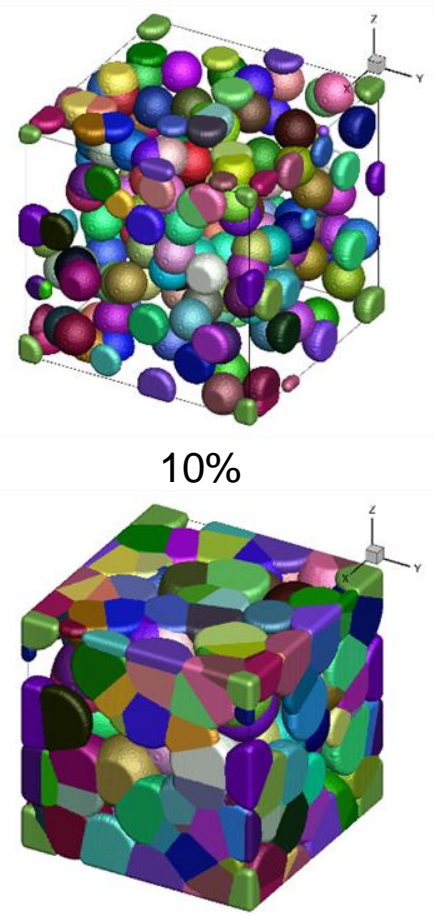

$70 \%$

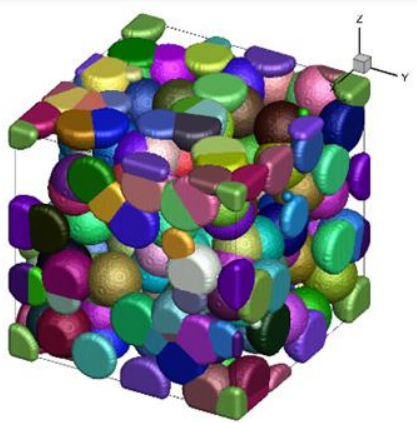

$30 \%$

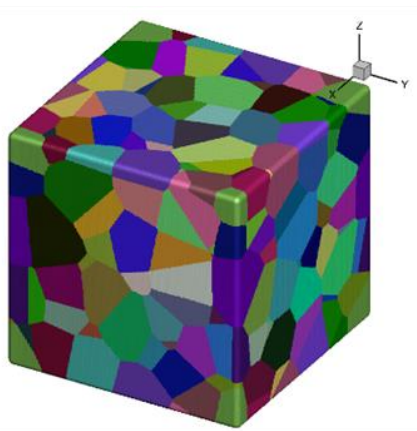

Final

Figura 2: Representação da microestrutura simulada de nucleação homogêneas com 150 núcleos com velocidades de crescimento iguais.

A sequência de micrografias mostra a microestrutura final da matriz e de cada um dos planos estudados. A Figura 3 se refere à simulação: matriz de $300^{3}, 150$ números de núcleos totais.

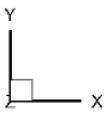

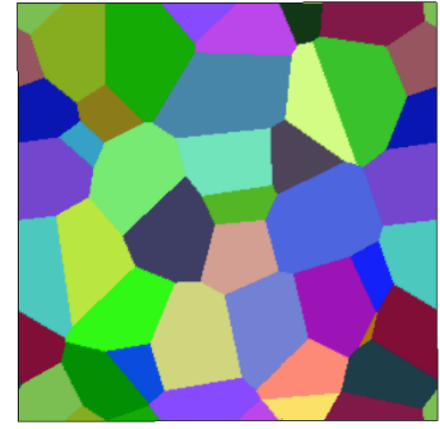

(a)

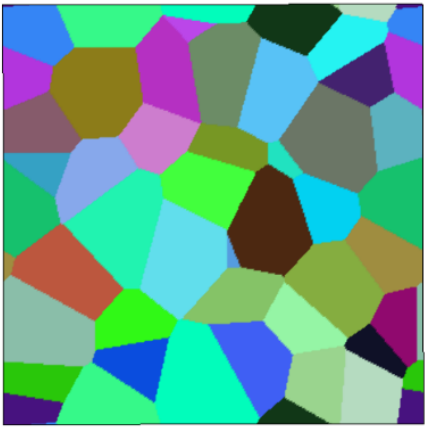

(b) 


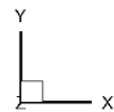

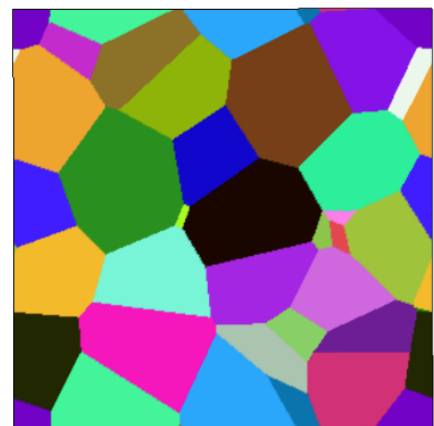

(c)

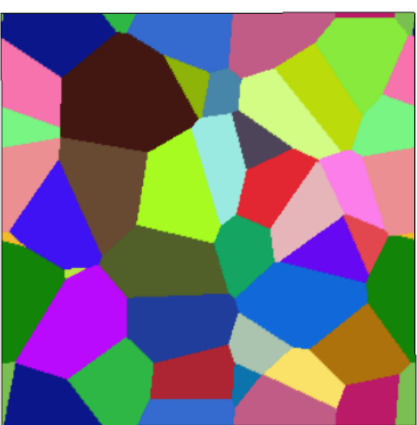

(d)

Figura 3: Fração volumétrica em quatro planos diferentes:(a) $z=0,1$, (b) $z=0,3$, (c) $z=0,7$, (d) $z=0,9$.

A Figura 4 apresenta a fração volumétrica $\left(V_{V}\right)$, área superficial por unidade de volume (Sv), a velocidade de reação $\mathrm{G}$ e o caminho microestrutural da nucleação homogênea.

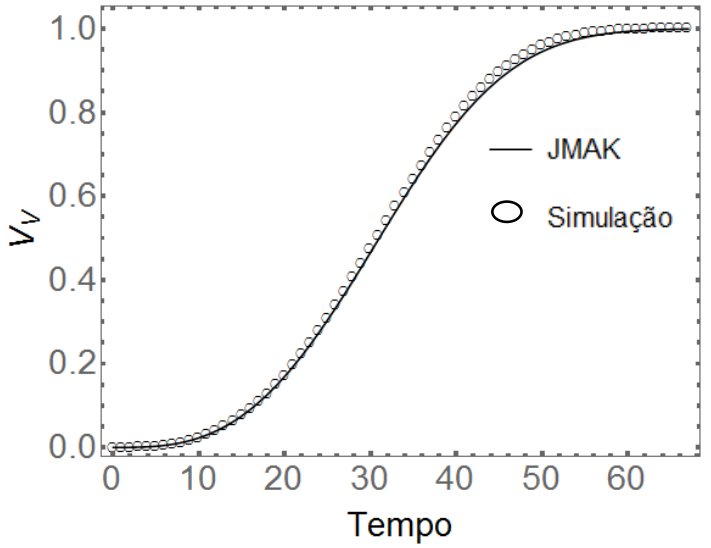

(a)

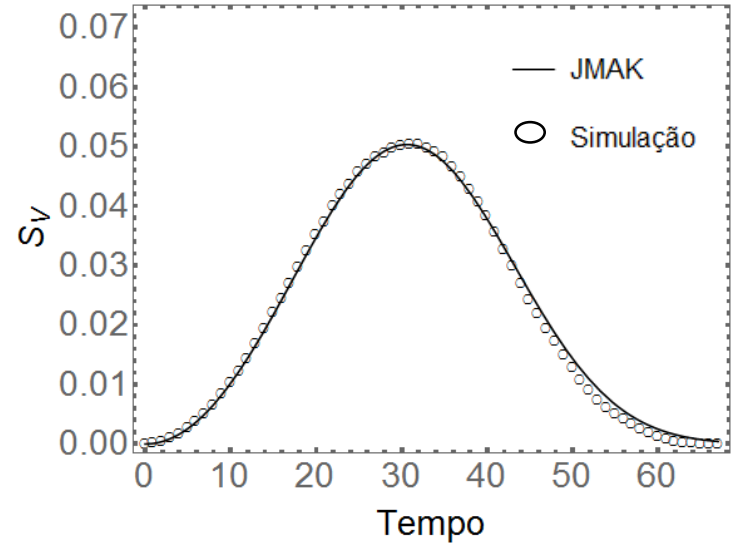

(b)

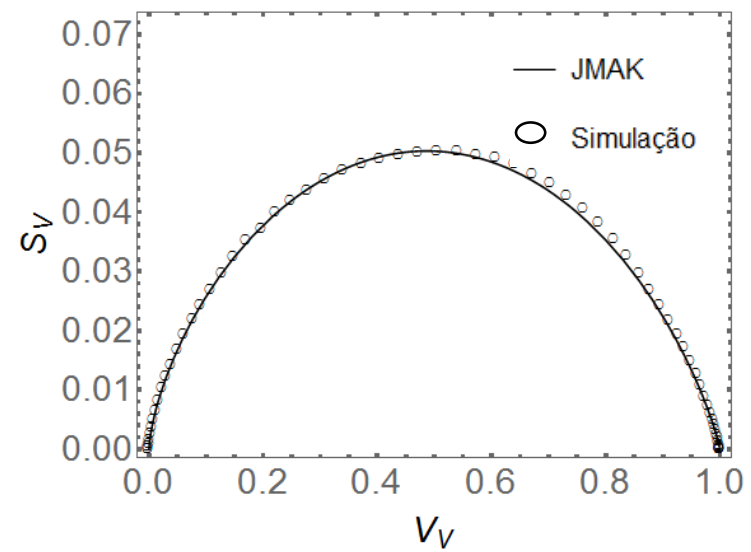

(c)

Figura 4: Resultados da nucleação homogênea (a) $V_{V} \times$ Tempo. (b) $S_{V} \times$ Tempo. (c) Caminho Microestrutural x Tempo 
Quando os núcleos estão distribuídos de forma aleatória na matriz o aumento do $V_{v}$ ocorre de maneira uniforme em toda a matriz. As microestruturas obtidas pela simulação computacional neste trabalho apresentam uma nucleação não uniforme na matriz distribuída de forma de um gradiente de deformação em única direção, podemos observar uma maior concentração de núcleos na parte superior da matriz.

A Figura 5 mostra evolução da nucleação não homogênea em uma matriz $300 \times 300 \times 300, \lambda(z)=596 z+2$, com um número de total 150 núcleos.
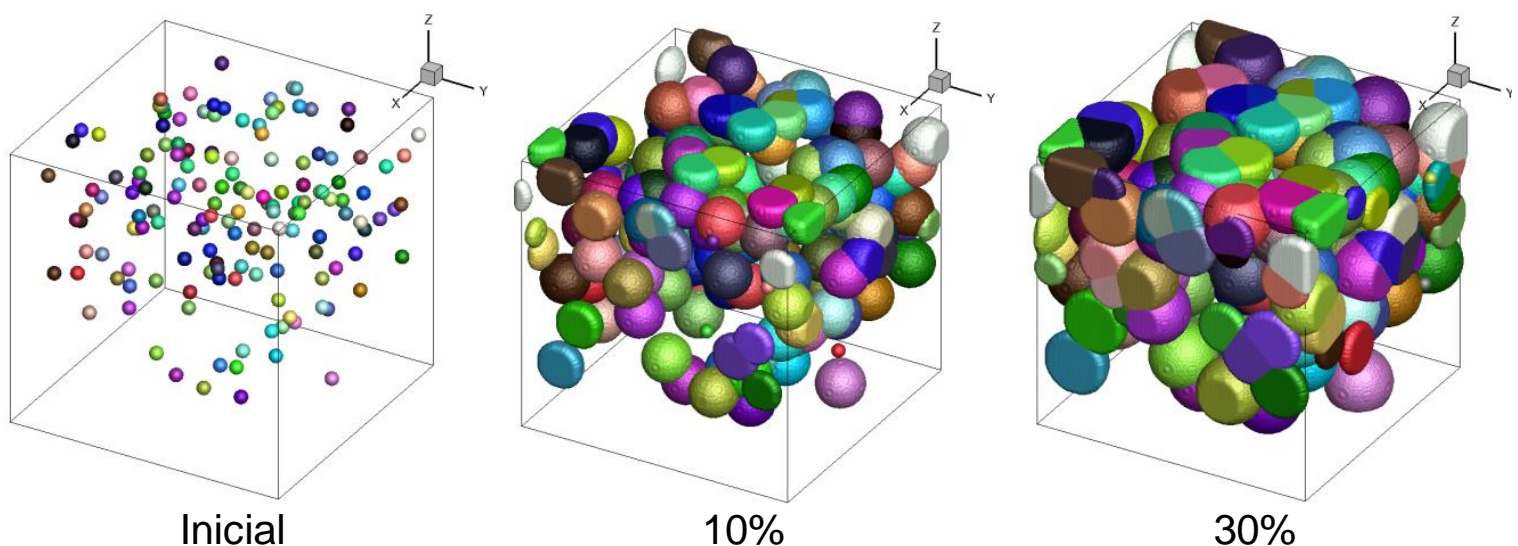

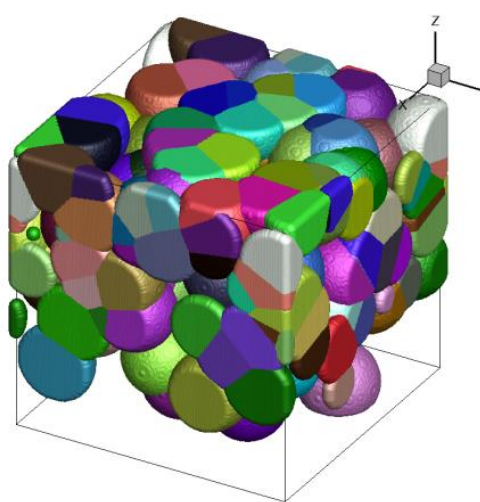

$50 \%$

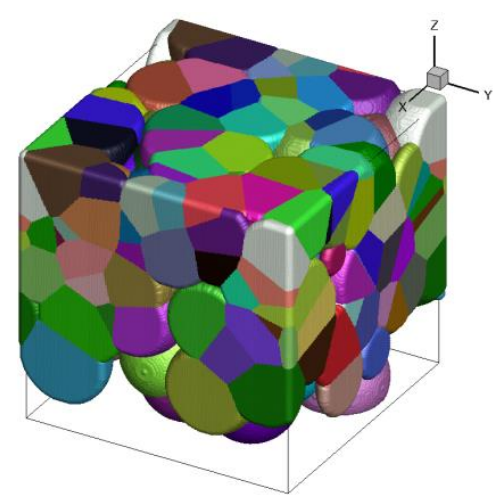

$70 \%$

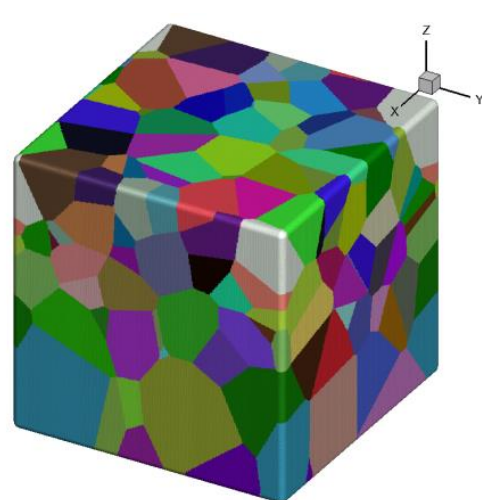

Final

Figura 5: Representação da microestrutura simulada de nucleação não homogêneas com 300 núcleos com velocidades de crescimento iguais.

A sequência de micrografias mostra a microestrutura final da matriz e de cada um dos planos estudados. A Figura 6 se refere à simulação: matriz de 300×300×300, $\mathrm{m}=596$ e $\mathrm{n}=2$ e 300 núcleos totais. 


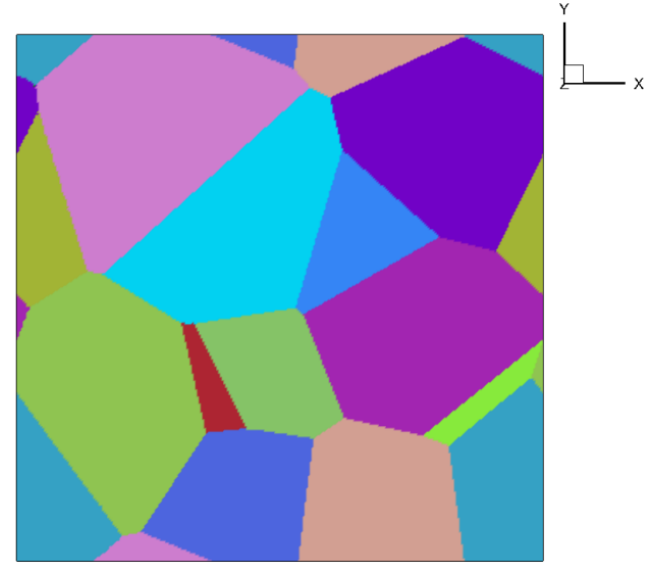

(a)

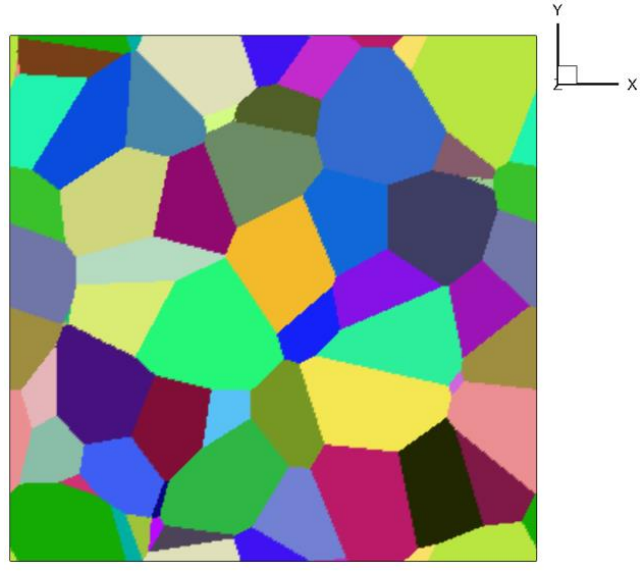

(c)

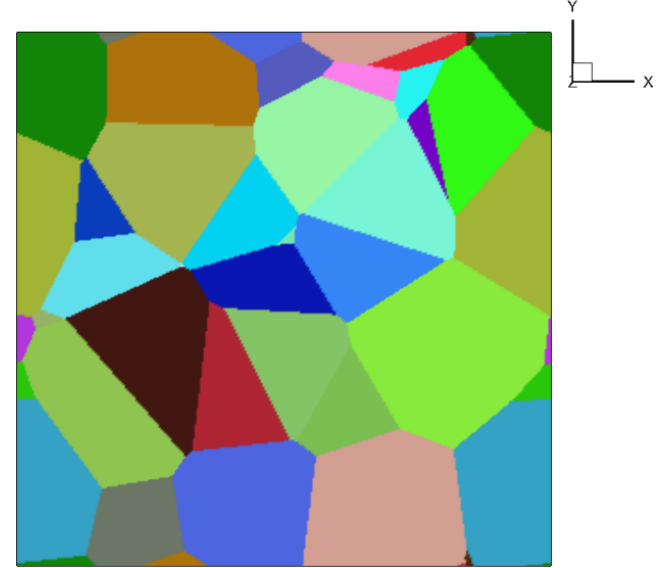

(b)

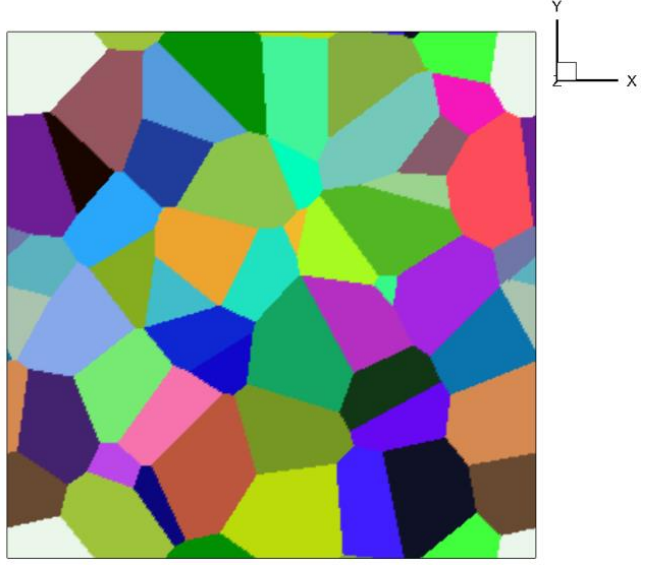

(d)

Figura 6: Fração volumétrica em quatro planos diferentes:(a) $z=0,1$, (b) $z=0,3$, (c) $z=0,7$, (d) $z=0,9$.

Observa-se que tamanho dos grãos são maiores nos planos $\mathrm{z}=0,1$ e $\mathrm{z}=0,3$ do que nos planos $z=0,7$ e $z=0,9$ isso se dá devido a quantidade de núcleos da reação depende da sua posição na matriz. Isso vai influenciar no tamanho dos grãos onde houver uma maior quantidade de núcleos os grãos serão menores do que nas áreas onde houver um menor número de núcleos.

A Figura 7 apresenta a fração volumétrica $V_{v}$, área superficial por unidade de volume, a fração volumétrica por planos e o caminho microestrutural na nucleação não homogênea.

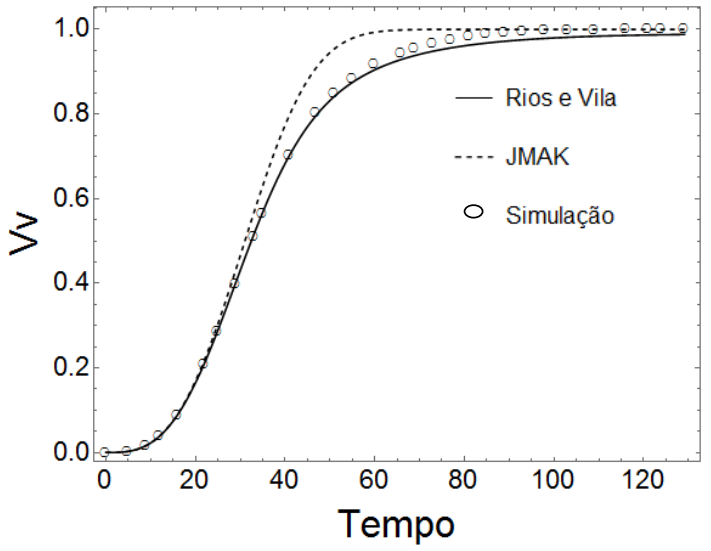

(a)

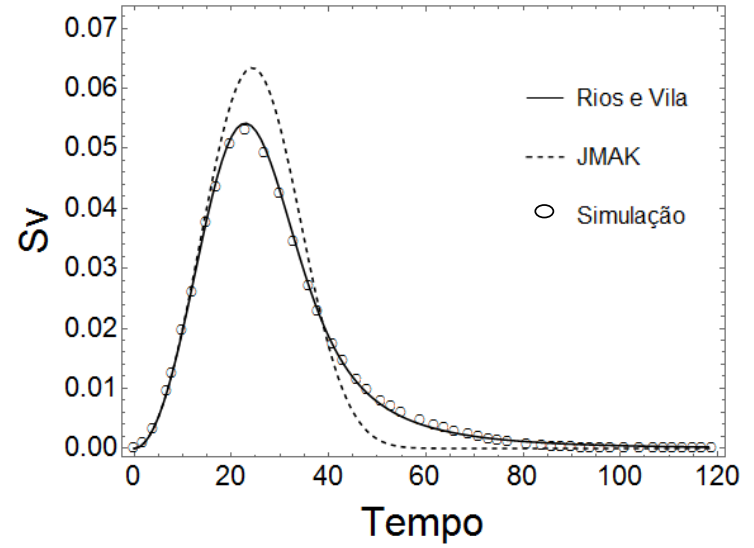

(b) 


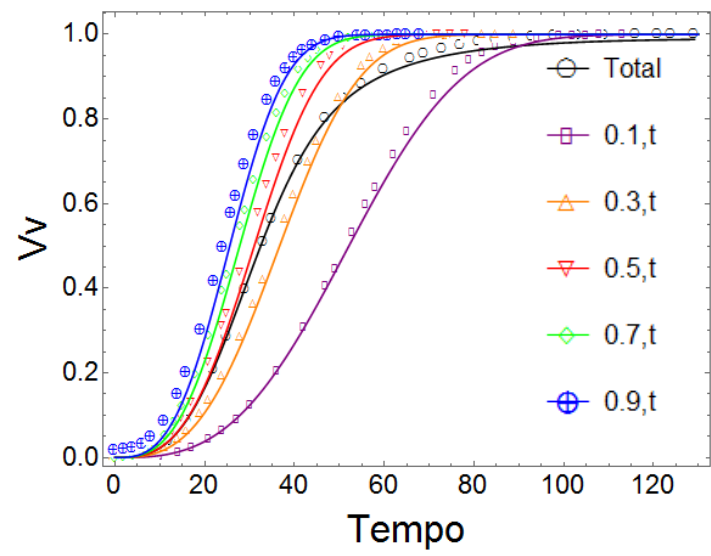

(c)

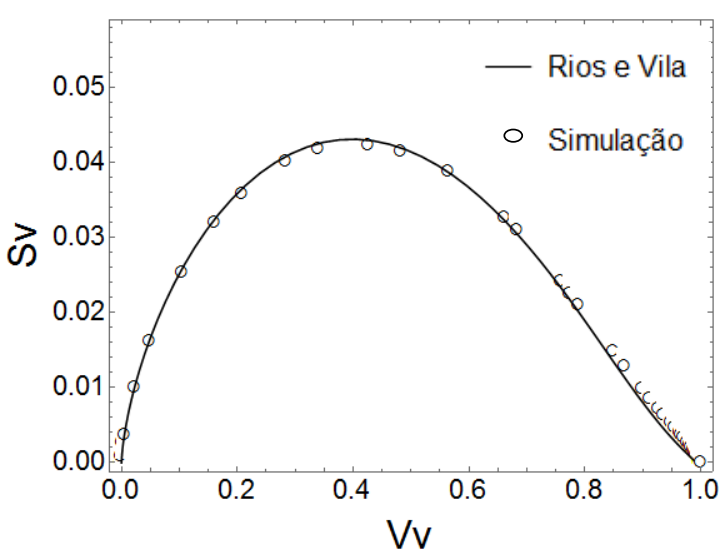

(d)

Figura 7: Resultados da nucleação não homogênea (a) $V_{V} \times$ Tempo. (b) $S_{V} \times$ Tempo. (c) $V_{v}$ por plano da matriz x Tempo. (d) Caminho Microestrutural x Tempo

$\mathrm{Na}$ nucleação não-homogênea $o$ aumento da $V_{V}$ não ocorrera de maneira uniforme mediante ao seu gradiente de deformação onde a região com menos quantidade núcleos vai determinar a cinética da reação. Dessa mesma forma gradiente de deformação influenciou no tempo de início da ocorrência da interferência entre as reações "impingement".

\section{CONCLUSÃO}

Os resultados obtidos permitem demonstrar a diferença entre a nucleação homogênea e não homogênea onde a influência da distribuição da posição dos núcleos inicia-se nas microestruturas completamente transformadas geradas pela simulação computacional e nos gráficos da evolução da transformação. $\mathrm{Na}$ nucleação homogênea apresentou uma microestrutura com os grãos com tamanho final uniforme em toda a matriz e sua a evolução da fração volumétrica ocorreu de uma forma uniforme em toda transformação. Na nucleação não homogênea gerou uma microestrutura onde o tamanho dos grãos finais foram dependente do seu gradiente de deformação nesse caso gerou na parte superior da matriz os grãos com um tamanho menor ao da região inferior da matriz e na evolução da fração volumétrica da transformação não ocorrer de forma uniforme na transformação nos planos superiores onde há uma maior quantidade de núcleos a fração volumétrica daquela região ocorre em tempo menor que as demais onde tem um menor concentração de núcleos.

\section{Agradecimentos}

Os autores agradecem a Universidade Federal Fluminense (UFF), CNPq, CAPES e FAPERJ. 


\section{REFERÊNCIAS}

1 JOHNSON, W. A. AND MEHL, R. F. Reaction kinetics in processes of nucleation and growth. Transactions Metallurgical Society, A.I.M.E, 1939, vol. 135 , p. $416-441$

2 AVRAMI, M. Kinetics of phase change I. General Theory. Journal of Chemical Physics, vol. 7, 1939, p.1103-1112.

3 AVRAMI, M. Kinetics of phase change II. Transformation - time relations for random distribution of nuclei. Journal of Chemical Physics, vol. 8, 1940, p. 212-224.

4 AVRAMI, M. Granulation, phase change, and microstructure kinetics of phase change. III. Journal of Chemical Physics, vol. 9, 1941, p. 177-184.

5 KOLMOGOROV, A. N. On the statistical theory of metal crystallization. Isvetiia Academii Nauk, SSSR-Seriia Matematicheskaia, 1937, vol. 1, p. 335-359.

6 RIOS, P. R. e VILLA, E. Transformation kinetics for inhomogeneous nucleation. Acta Materialia, v. 57, n. 4, p. 1199-1208, 2009.

7 ASSIS, T.C.S., Modelamento Numérico de Nucleação e Crescimento via Autômato Celular, Tese de doutorado, UFF - Volta Redonda., 2011.

8 ALVES, A.L.M. Simulação computacional de reações com nucleação e crescimento de fases simultâneas e sequenciais pelo método do cone causal, Dissertação de mestrado, UFF-Volta Redonda, 2015.

9 COSTA, M. F. B., Simulação computacional de transformações não homogêneas por nucleação e crescimento, Dissertação de mestrado, UFFVolta Redonda, 2017. 